\title{
Preservação dos Ovários em Cirurgia Radical para Câncer do Colo Uterino
}

\author{
Ovarian Preservation in Radical Surgery for Cervical Cancer
}

Sabas Carlos Vieira, André Gonçalves da Silva, Lia Rachel Gomes do Vale, Murilo Moura Lima, João Batista Mendes Teles

\begin{abstract}
RESUMO
Objetivo: avaliar a função ovariana em pacientes com câncer do colo uterino, que se submeteram a histerectomia radical com preservação dos ovários.

Métodos: foram analisadas retrospectivamente pacientes com câncer do colo uterino, submetidas a histerectomia radical com preservação dos ovários na Clinica Ginecológica do Hospital São Marcos-SPCC, de abril de 1998 a outubro de 2001, com avaliação dos sintomas de privação estrogênica (fogachos, vagina seca) e mensuração dos niveis de FSH pósoperatórios. Os dados foram analisados pelo teste de Pearson.

Resultados: os niveis de FSH foram mensurados em 42 pacientes; destas, 33 (78,5\%) apresentaram niveis normais de FSH no pós-operatório (menores que $30 \mathrm{mU} / \mathrm{mL}$ ); os valores variaram entre 1,2 e 132,44 $\mathrm{mU} / \mathrm{mL}$ (mediana de 21,05 $\mathrm{mU} / \mathrm{mL}$ ). Das nove pacientes com niveis elevados de FSH, cinco (55,6\%) haviam sido submetidas a radioterapia pós-operatória ( $p<0,0001)$. Não houve associação entre a idade das pacientes e os niveis de FSH ( $p=0,33)$. Cistos ovarianos funcionais ocorreram em quatro pacientes (7,7\%). Uma paciente apresentou recidiva da lesão na cúpula vaginal e metástase para o couro cabeludo, evoluindo para óbito. Conclusão: observou-se preservação da função ovariana em 78,5\% das pacientes. A transposição ovariana foi inadequada para preservar sua função em pacientes submetidas à radioterapia pós-operatória. Não se observou associação entre idade e níveis de FSH no pósoperatório.
\end{abstract}

PALAVRAS-CHAVE: Colo uterino: câncer. FSH. Falência ovariana.

\section{Introdução}

O câncer do colo uterino é sério problema de saúde pública na América Latina, sendo a primeira causa de morte na população feminina em idade reprodutiva ${ }^{1}$. O tratamento de escolha para casos de câncer do colo uterino invasivo em estágios iniciais é a histerectomia radical com salpingooforectomia bilateral. Entretanto, a preservação dos ovários em pacientes jovens submetidas à cirurgia radical para câncer do colo uterino apresenta manutenção da função ovariana entre 70 e $83 \%$ dos casos, bem como baixa

\footnotetext{
Hospital São Marcos - Teresina - PI

Correspondências:

André Gonçalves da Silva

Rua Fontes Ibiapina 1571 - Ininga

64049-780 - Teresina - PI

e-mail: ags-md@ig.com.br
}

morbidade associada ${ }^{2-4}$. Tendo em vista que parcela significativa dos casos de câncer do colo uterino ocorre em mulheres jovens, a preservação dos ovários deve sempre ser oferecida à paciente, visto que a ooforectomia bilateral implicará sintomatologia vasomotora e gênito-urinária associada ao climatério e complicações mórbidas como a osteoporose. Além disso, a reposição estrogênica nas pacientes submetidas a ooforectomia implica comprometimento importante do orçamento familiar, devido ao baixo nivel socioeconômico da maioria das pacientes com câncer do colo uterino ${ }^{5,6}$.

Este estudo tem como objetivo a avaliação da função ovariana em pacientes com câncer do colo uterino, que se submeteram a histerectomia radical com manutenção dos ovários. 


\section{Pacientes e Métodos}

Foram analisados retrospectivamente os prontuários das pacientes com diagnóstico de câncer invasivo do colo uterino, submetidas a histerectomia radical com preservação ovariana, tratadas na Clínica de Ginecologia e Mastologia do Hospital São Marcos-Sociedade Piauiense de Combate ao Câncer (SPCC), no periodo de abril de 1998 a outubro de 2001 . Os critérios de inclusão foram: idade inferior a 45 anos, ciclos menstruais regulares, ausência de sintomas de hipoestrogenismo (fogachos, vagina seca) e ovários macroscopicamente normais ao ato cirúrgico. Todas as pacientes foram submetidas a histerectomia radical à Piver 3, com linfadenectomia pélvica bilateral, após consentimento verbal quanto à preservação ovariana. Os ovários preservados foram fixados nas goteiras parieto-cólicas homolaterais no nivel da bifurcação da aorta e marcados com clipes metálicos número 200, sempre se observando a posição adequada do pedículo vascular ovariano no sentido de evitar torção e/ou tração. O peritônio pélvico foi deixado aberto e a drenagem pélvica foi realizada com dreno de sucção a vácuo posicionado na cavidade pélvica, com exteriorização por contra-abertura na parede abdominal. Radioterapia foi realizada nas pacientes que apresentaram pelo menos um dos seguintes critérios: linfonodos comprometidos, margens comprometidas, margem vaginal livre de doença menor que $0,5 \mathrm{~cm}$, invasão linfática e recidiva loco-regional.

O seguimento das pacientes nos primeiros dois anos foi realizado a cada 4 meses e a partir de então a cada 6 meses. No retorno as pacientes eram submetidas a exame ginecológico com coleta de material da cúpula vaginal para citologia oncótica. Ultra-sonografia foi realizada nas pacientes que apresentaram queixa de dor abdominal em qualquer momento do seguimento. Os niveis de FSH foram mensurados pelo menos uma vez no seguimento inicial.

Foram analisados os seguintes dados: idade, tipo histológico, estadiamento, sintomas de privação estrogênica no pré e pós-operatório, ciclos menstruais no pré-operatório e mensuração do nivel sérico de FSH no pós-operatório. Utilizouse o teste de Pearson para análise estatística, com nivel de significância de $5 \%$.

O estudo foi aprovado pelo Comitê de Ética em Pesquisa da Universidade Federal do Piauí.

\section{Resultados}

Foram submetidas à cirurgia de WertheimMeigs, com preservação bilateral dos ovários, 42 pacientes, com idade média de 36 anos, variando entre 26 e 44 anos. Todas as pacientes encontravam-se em estádio Ib1 da FIGO. O número de linfonodos retirados variou entre 9 e 32, com uma média de 17,2. O tipo histológico predominante foi o carcinoma espinocelular, com 38 casos $(90,4 \%)$. Em todas as pacientes as margens parametriais e vaginais estavam livres de comprometimento neoplásico ao exame histopatológico (Tabela 1).

Tabela 1 - Pacientes submetidas a histerectomia radical com preservação ovariana no período de abril de 1998 a outubro de 2001.

Idade (média) 36

Estadiamento da FIGO Ib1 (número de casos)

Tipo histológico (número de casos)

Carcinoma epidermóide

Adenocarcinoma

Radioterapia 9

Do total de pacientes do estudo, observaram-se valores normais de FSH (inferior a 30 $\mathrm{mU} / \mathrm{mL}$ ) em 33 pacientes $(78,6 \%)$. Os valores de FSH encontrados variaram entre 1,2 e 132,44 $\mathrm{mU} / \mathrm{mL}$, com média de 21,05 mU/mL. Das nove $(21,4 \%)$ pacientes que apresentaram niveis elevados, cinco $(55,6 \%)$ haviam sido submetidas à radioterapia pós-operatória $(\mathrm{p}<0,0001)$ (Tabela 2$)$. A idade não apresentou associação positiva com os níveis pós-operatórios de FSH ( $p=0,33$, índice de Pearson $=0,93$ ).

Tabela 2 - Resultados das dosagens de FSH

\begin{tabular}{lcccc}
\hline Parâmetro & \multicolumn{2}{c}{ FSH $<\mathbf{3 0} \mathbf{~ m U / m L}$} & \multicolumn{2}{c}{ FSH $>\mathbf{3 0} \mathbf{~} \mathbf{~ U / m L}$} \\
\hline Radioterapia & Sim & Não & Sim & Não \\
& $1(3 \%)$ & $32(97 \%)$ & $5(55,6 \%)$ & $4(44,4 \%)$ \\
\hline$n=42$ & & & &
\end{tabular}

Observou-se a ocorrência de cistos ovarianos funcionais benignos à ultra-sonografia em quatro pacientes $(7,7 \%)$. Com período de seguimento mediano de 16,4 meses, variando entre quatro e 33 meses, observou-se apenas um óbito. Esta paciente apresentou recidiva da lesão tumoral na cúpula vaginal e metástases no couro cabeludo. As demais pacientes estão vivas e sem evidência de doença em atividade ou sintomas de hipoestrogenismo. 


\section{Discussão}

Neste estudo, observou-se manutenção da função ovariana em $78,6 \%$ das pacientes submetidas ao procedimento cirúrgico, dados que condizem com os achados na literatura ${ }^{4}$. As taxas de sucesso na preservação da função dos ovários parecem estar relacionadas basicamente a dois fatores: a realização de radioterapia pós-operatória e a idade das pacientes. Desse modo, tem-se preconizado a preservação dos ovários em pacientes com menos de 40 anos ${ }^{7}$.

O temor de metástases ovarianas em lesões escamosas $(<1 \%)$ ou adenocarcinoma cervical uterino $(1,4 \%)^{8,9}$, não constitui indicação para remoção de ovários de aparência macroscopicamente normal durante a cirurgia em pacientes jovens submetidas a histerectomia radical, desde que estas apresentem ciclos menstruais regulares e ausência de sinais e/ou sintomas compativeis com privação estrogênica ${ }^{2}$. Nos relatos de comprometimento ovariano, a extensão endometrial ou para o corpo uterino foi encontrada, sugerindo a possibilidade de extensão direta da neoplasia para o ovário ${ }^{8}$.

Com o conhecimento das conseqüências da privação estrogênica na qualidade de vida (sintomatologia vasomotora e urogenital), bem como no desenvolvimento de complicações mórbidas (osteoporose, doença cardiovascular) ${ }^{10}$, e tendo também conhecimento de que parcela significativa das pacientes acometidas por essa neoplasia pertence a classes sociais mais baixas (e, portanto, sem condições de manterem reposição estrogênica em longo prazo), esta opção deve ser considerada pelos médicos, visto que se relaciona a melhor qualidade de vida para as pacientes, com baixos níveis de morbidade ${ }^{11}$.

Pete e Bosze ${ }^{12}$ avaliaram 65 pacientes com carcinoma de colo uterino em estádios iniciais tratadas com histerectomia radical e preservação ovariana, e, considerando como critério diagnóstico de falência ovariana a mensuração de três valores de FSH acima de $30 \mathrm{mU} / \mathrm{mL}$, encontraram falência ovariana em duas pacientes. Nenhuma paciente apresentou recorrência da doença. Três pacientes foram submetidas a salpingooforectomia 6, 11 e 24 meses após a histerectomia radical, por apresentarem lesões anexiais, todas benignas ao exame histopatológico.

Morice et al. ${ }^{4}$, em estudo com 107 pacientes com idade média de 33 anos submetidas a histerectomia radical com preservação ovariana (com fixação nas goteiras paracólicas), conseguiram a manutenção da função ovariana em todas as pacientes submetidas exclusivamente a procedi- mento cirúrgico, contra $90 \%$ entre as pacientes tratadas com braquiterapia vaginal pós-operatória e $60 \%$ das tratadas com radiação externa e braquiterapia pós-operatória. Tais dados indicaram maior risco de falência ovariana associada à prática de radioterapia externa pós-operatória. Já Ishii et al. ${ }^{3}$ avaliando 33 pacientes submetidas a histerectomia radical com preservação ovariana, observaram falência ovariana em 5 de 21 pacientes até 39 anos, contra 10 de 12 pacientes com mais de 40 anos; analisando estes dados por meio de análise de regressão múltipla, observaram correlação significativa entre disfunção ovariana e idade superior a 40 anos.

Em nosso estudo, observamos predomínio dos casos de falência ovariana entre as pacientes submetidas à radioterapia, dado que confirma os relatos da literatura. Este fato pode ser devido a uma possivel fixação inadequada dos ovários fora da pelve. O estudo não demonstra associação positiva entre a idade das pacientes e os niveis pósoperatórios de FSH, possivelmente devido ao pequeno tempo de seguimento, pois se esperava que as pacientes que apresentassem maior idade viessem a apresentar falência ovariana mais precocemente que as pacientes mais jovens. Outro fator que pode estar implicado na ausência de correlação entre a idade e os niveis pós-operatórios de FSH é o pequeno número de casos do estudo.

\section{ABSTRACT}

Purpose: to assess ovarian function in patients with cervical cancer following radical hysterectomy with ovarian preservation.

Methods: we retrospectively analyzed patients with cervical carcinoma, submitted to radical hysterectomy with ovarian preservation at the Gynecologic Clinic of the São Marcos Hospital-SPCC, from April 1998 to October 2001, with evaluation of symptoms of estrogenic deprivation (flushing, dry vagina) and the measurement of FSH levels after surgery. All data were analyzed using the Pearson test.

Results: FSH levels were measured in 42 patients; of these, $33(78.5 \%)$ patients had normal FSH levels (below $30 \mathrm{mU} /$ $\mathrm{mL}$ ). The median level was $21.05 \mathrm{mU} / \mathrm{mL}$ (range 1.2-132.44 $\mathrm{mU} / \mathrm{mL}$ ). Five $(55.6 \%)$ of the nine patients with high $\mathrm{FSH}$ levels had received postoperative radiotherapy $(p<0.0001)$. There was no correlation between postoperative FSH levels and age over 40 years $(p=0.33)$. Benign ovarian cysts occurred in four patients $(7.7 \%)$. One patient presented recurrence of the lesion in the vaginal dome and metastasis to the scalp, and died.

Conclusion: in $78.5 \%$ of the patients, ovarian function was preserved. Ovarian transposition was inadequate to preserve 
ovarian function in patients who underwent postoperative radiotherapy. There was no correlation between age and postoperative FSH levels.

KEY WORDS: Cervical cancer. FSH. Ovarian failure.

\section{Referências}

1. Novoa Vargas A, Echegollen Guzmán A. Epidemiología del cáncer de cérvix em Latinoamérica. Ginecol Obstet Mex 2001; 69:243-6.

2. Buekers TE, Anderson B, Sorosky JI, Buller RE. Ovarian function after surgical treatment for cervical cancer. Gynecol Oncol 2001; 80:85-8.

3. Ishii K, Aoki Y, Takakuwa K, Tanaka K. Ovarian function after radical hysterectomy with ovarian preservation for cervical cancer. J Reprod Med 2001; 46:347-52.

4. Morice P, Juncker L, Rey A, El-Hassan J, Haie-Meder C, Castaigne D. Ovarian transposition for patients with cervical carcinoma treated by radiosurgical combination. Fertil Steril 2000; 74:743-8.

5. Giacalone PL, Laffargue F, Benos P, Dechaud H, Hedon B. Successful in vitro fertilization-surrogate pregnancy in a patient with ovarian transposition who had undergone chemotherapy and pelvic irradiation. Fertil Steril 2001; 76:388-9.
6. Olejek A, Wala D, Chimiczewski P, Rzempoluch J. Hormonal activity of transposed ovaries in young women treated for cervical cancer. Gynecol Endocrinol 2001; 15:5-13.

7. Morice P, Castaigne D, Haie-Meder C, et al. Laparoscopic ovarian transposition for pelvic malignancies: indications and functional outcomes. Fertil Steril 1998; 70:956-60.

8. Sutton GP, Bundy BN, Delgado G, et al. Ovarian metastases in stage IB carcinoma of cervix: a Gynecology-Oncology Group of Study. Am J Obstet Gynecol 1992; 166:50-3.

9. Yamamoto R, Okamoto K, Yukiharu T, et al. Study of risk factors for ovarian metastases in stage IbIIIb cervical carcinoma and analysis of ovarian function after a transposition. Gynecol Oncol 2001; 82:312-6.

10.Vieira SC, Coelho FRG, Mourão Netto M. Risco de câncer de mama e endométrio em vigência de reposição hormonal na pós-menopausa: um problema para ginecologistas e cancerologistas. Acta Oncol Bras 2000; 20:32-7.

11.Sipos N, Szantho A, Csapo Z, Balega J, Demeter A, Papp Z. Transposition of ovaries during radical hysterectomy for cervical cancer. Orv Hetil 2002; 143:189-92.

12.Pete I, Bosze P. The fate of the retained ovaries following radical hysterectomy. Eur J Gynaecol Oncol 1998; 19:22-4.

Recebido em: 17/9/2002 Aceito com modificações em: 26/11/2002

\section{A FEBRASGO DESEJA AOS SEUS ASSOCIADOS UM

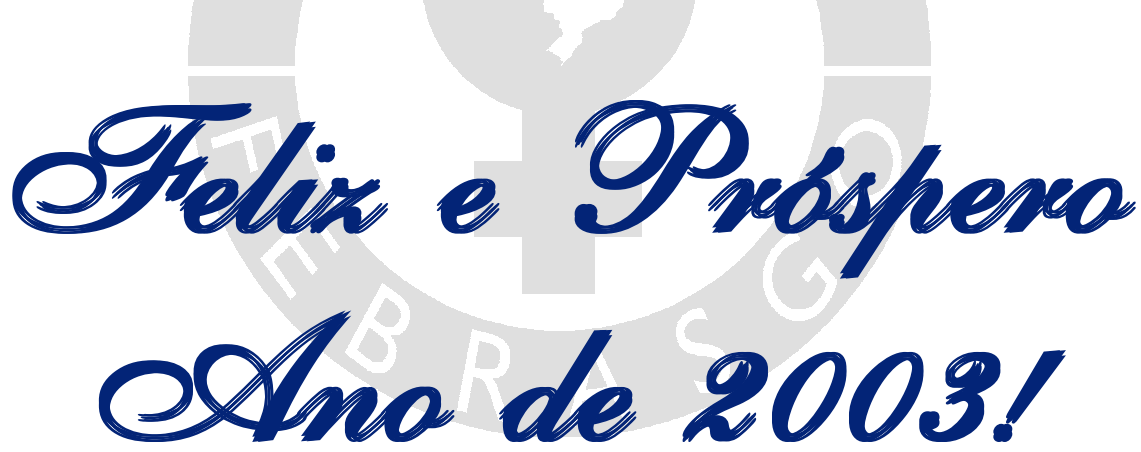

